Draft Version OCTOBer 1, 2019

Typeset using $\mathrm{LAT}_{\mathrm{E}} \mathrm{X}$ twocolumn style in AASTeX62

\title{
A LOOK AT PHASE SPACE INTERMITTENCY IN MAGNETIZED PLASMA TURBULENCE
}

\author{
Bogdan Teaca, ${ }^{1}$ Alejandro Bañón Navarro, ${ }^{2}$ Daniel Told,${ }^{2}$ Tobias Görler, ${ }^{2}$ Gabriel Plunk, ${ }^{3}$ David Hatch, ${ }^{4}$ \\ AND FRANK JENKO ${ }^{2}$ \\ ${ }^{1}$ Applied Mathematics Research Centre, Coventry University, Coventry CV1 5FB, United Kingdom \\ ${ }^{2}$ Max-Planck-Institut für Plasmaphysik, Boltzmannstr. 2, D-85748 Garching, Germany \\ ${ }^{3}$ Stellarator Theory, Max Planck Institute for Plasma Physics, Greifswald, Germany \\ ${ }^{4}$ Institute for Fusion Studies, The University of Texas at Austin, Austin, Texas, USA
}

\begin{abstract}
In a proton-electron magnetized plasma, we analyze turbulence at kinetic scales captured by a gyrokinetic formalism. The interval of scales spans the range between the proton and the electron gyroradii, while the use of the proper mass ratio between the two species ensures a realistic scale separation for the nonlinear couplings. The simulation is pertinent to astrophysical conditions, employing a straight field line magnetic geometry for the guide field, a plasma $\beta$ of one and a temperature ratio between the two species of unity. We investigate the intermittency of the distribution functions in the perpendicular direction, measured over the phase space as a way to account for the velocity space structures generated via Landau damping as well as for the nonlinear spatial mixing (i.e. the turbulent cascade). The analysis makes use of a Hermite decomposition in the parallel velocity. Electron structures are found to be strongly intermittent compared to weakly intermittent protons. Moreover, we find evidence linking intermittency with phase mixing and electron Landau damping, as intermittent electron structures also exhibiting strong parallel velocity structures.
\end{abstract}

\section{INTRODUCTION}

Turbulence in astrophysical plasma occurs over a wide range of spatial scales Bruno \& Carbone (2013); Kiyani et al. (2015) ( $\ell$ ). While large scale dynamics can be captured by fluid approximations Zhou et al. (2004), the physics of turbulence on scales comparable to the proton (ion) gyroradius and smaller requires a kinetic description Marsch (2006); Schekochihin et al. (2009); Howes et al. $(2008,2011)$ and as such, velocity space interactions need to be considered. Moreover, the departure of turbulence from self-similarity (i.e. scale invariance of the dynamics) leads to intermittency, which impacts simultaneously the locality of energetic interactions between scales Teaca et al. (2017) and the formation of structures in real space Frisch (1995). The emergence of inhomogeneous structures is known to affect the transport of particles and heat in turbulent media, but at the kinetic level, as we will explore in the current work, they can affect the balance between dynamics in position space and velocity space.

The solar wind is the best example of this overall scenario, as a mean free path on the order of an $\mathrm{AU}$ requires

Corresponding author: Bogdan Teaca

bogdan.teaca@coventry.ac.uk us to investigate the problem from the perspective of a collisionless plasma. This means that the sink of turbulent fluctuations cannot be modeled via simple viscous terms and requires a kinetic description in a (positionvelocity) phase space, including the use of appropriate collision operators. In fact, in the solar wind, the magnetohydrodynamic (MHD) turbulent cascade is found to give way to a kinetic cascade at scales below the proton gyroradius. The kinetic range, traditionally referred to as the dissipation range, exhibits nonlinear interactions that cannot be neglected as in a viscous dissipation range. In this range, the dynamics are further complicated by the need to incorporated velocity space effects (i.e. phase mixing).

While the name dissipation range in the solar wind is not emblematic of the underlying nonlinear dynamics, it is appropriate from a phenomenological perspective. In this range we observe the general thermalization of the plasma fluctuations (thermalization should be seen in the broader sense, as the way phase space fluctuations reach steady state through the action of collisions, rather than classical thermodynamic equilibrium). The general thermalization of plasma is related to the heating problem of the solar wind Bruno \& Carbone (2013), both in an entropy production sense and in the sense of 
the mechanism that leads to a temperature increase and its anisotropization.

As the importance of kinetic turbulence in the analysis of astrophysical plasma is evident, the question of intermittency and its interplay within the encompassing phase space emerges. Turbulence is typically associated with the development of localized structures, such as eddies, filaments and sheets, with the energy at small scales being contained in a few energetic structures Zhdankin et al. (2015). Intermittency can thus be seen as the tendency of small scales to be less volume filling than larger scales Frisch (1995) and we will show this aspect in relation to the magnetic field. At kinetic scales, the dissipation of electromagnetic fluctuations is found to occur in highly localized current sheets by a series of numerical studies Wan et al. (2012, 2015); Karimabadi et al. (2013); Tenbarge \& Howes (2013) and satellite observations of the solar wind Perri et al. (2012b,a); Osman et al. (2012b,a, 2014b); Sundkvist et al. (2007). At the same time, particlewave resonance is known to exchange energy between the electromagnetic fields and the particle distributions Tenbarge \& Howes (2013); Cerri et al. (2016); Klein et al. (2017); Cerri et al. (2018); Kawazura et al. (2019); Meyrand et al. (2019); Chen et al. (2019), affecting the generation of velocity structures in phase space. Analyzing the intermittency and the velocity structures of the distribution functions is crucial for assessing the correct route to collisional dissipation and the balance that emerges between linear and nonlinear dynamics at kinetic levels.

The tendency of plasmas to become magnetized at smaller scales (and we emphasize that in the solar wind this is a tendency more than the realization of a strong magnetization) further amplifies phase mixing dynamics, as the free streaming of particles along magnetic field lines smooths out charge density fluctuations via Landau damping and generates finer velocity space structure in the process. The formation of intermittent spatial structure at these kinetic scales can in turn impact the location where smaller scales are generated in velocity space and represents the broader interest of the current work. As this is a general problem in collisionless magnetized plasma that can affect the temperature ratio between plasma species, it can impact spectroscopic measurements and estimates of distant objects (e.g. accretion disks, exoplanets). At kinetic scales, intermittency can play a much more important role than in fluid turbulence, as localized patches can support different regimes of phase space dynamics than the surrounding turbulent background. This would imply that the mechanism that leads to heating and temperature disparity between species has a strong local character and cannot be captured fully by homogenous estimates.

\subsection{A brief overview of intermittency and kinetic scales dynamics}

Dynamically, the energy exchanges between scales (i.e. the energy cascade) and the phenomenon of intermittency have the same underlying cause: nonlinear interactions. The nonlinear mixing can lead to phase correlations between fluctuations, which generates intermittency. In Fourier space, which provides a natural projection of the turbulent dynamics on a hierarchy of scales $(k \sim 1 / \ell)$, these correlations are contained in the complex phases of the Fourier modes. The way the phases are correlated with each other determines how the energy of a mode is distributed in real space, either in a statistically uniform manner or in a few localized patches. A random or any uncorrelated distribution of phases will show no spatial intermittency regardless of the shape of the spectrum. Traditionally, structure functions defined on field increments are employed for the study of intermittency. However, in our current work we will make use of scale functions for the analysis of intermittency, which are based on the Fourier space representation of scales. While this is not a widespread approach, we cannot claim novelty as this is an expansion on the idea presented in Ref. [Frisch 1995] for the analysis of the kurtosis.

For kinetic turbulence and its rigorous gyrokinetic (GK) Brizard \& Hahm (2007); Krommes (2012) limit in strongly magnetized plasmas, the distribution functions of the plasma species represent the dynamical quantities of interest. The role of the self-consistent electromagnetic fields, obtained from moments of the particles' distributions, is to mediate the nonlinear interactions between structures in the distribution functions Tatsuno et al. (2009). This represents the underlying mechanism for the development of intermittency at the kinetic level. The kinetic dynamics occur in a position-velocity phase space Schekochihin et al. (2008) involving couplings between velocity space structures Hammett et al. (1992); Plunk \& Tatsuno (2011); Plunk et al. (2010) in addition to those between spatial scales Navarro et al. (2011). Unlike in fluid representations, at the kinetic level there is no immediately evident mechanism for the direct correlation of the phases of the fields. The intermittency exhibited by the fields can be seen as being inherited from the dynamics of the distribution functions. This interpretation can be seen as the most robust one as it holds true regardless of the kinetic limits or models employed Eyink (2018). 
Alternatively, a 'hybrid' approach can be considered, where, following an appropriate Hermite (and Laguerre) decomposition of the distribution functions into a series of velocity moments, similar to Zocco \& Schekochihin (2011); Hatch et al. (2014); Parker \& Dellar (2015); Kanekar et al. (2015); Mandell et al. (2018), the equations for the Alfvénic fluctuations (and density fluctuations of the plasma) can be explicitly tracked. The nonlinear self-interaction between Alfvénic like fluctuations are now explicit in form. The reduced distribution function (basically the leftover of the original distribution once the first few moments have been subtracted) are seen as being passively advected by the Alfvénic fluctuations, e.g. Kunz et al. (2016); Meyrand et al. (2019). While different numerical schemes may be preferred for different implementations, we see the two approaches as being effectively equivalent and a reader should not be confused by the 'hybrid' label nor discouraged by it. We mention this to emphasize that the conceptual difference lies in the physical interpretation, as the latter approach tracks separately Alfvénic fluctuations (richer in phenomenological meaning) from the rest of the arbitrarily large number of Hermite modes, while the former approach interprets the dynamics from the perspective of phase space interactions (useful in the mathematical description of kinetic systems).

\subsection{Article structure}

In this work, we study intermittency of turbulence at kinetic scales, accounting for phase space structures in the GK limit. After we introduce the simulation details in Section 2, we start our study in Section 3 by defining our diagnostics and look at the intermittency in the perpendicular direction of the familiar magnetic fluctuations as a way to build confidence in the approach. The importance of velocity structures are showcased in Section 4 and the Hermite representation for the parallel velocity is introduced in Section 5. We then compute in Section 6 scaling functions directly on the proton (ion) and electron distributions, which account for velocity space fluctuations and their mixing in phase space. In Section 7, we discuss the intermittent electron structures from the perspective of linear phase mixing, indirectly showing that smaller structures in the parallel velocity are generated in the high intensity spatial structures. We end in Section 8 by looking at the dependence of the perpendicular spatial cascade with the scale size of the parallel velocity structures, determined through the use of scale filtering in Hermite space, before drawing our conclusions in Section 9.

\section{SIMULATION DETAILS}

In this study we use gyrokinetic simulations of magnetized proton-electron plasmas. This formalism assumes low frequencies (compared to the ion, here proton, cyclotron frequency) and small fluctuation levels to remove the particles' fast gyro-motion, effectively reducing the relevant phase space to five-dimensions Brizard \& Hahm (2007); Howes et al. (2006). While it neglects cyclotron resonance, gyrokinetics captures the crucial dynamics Told et al. (2016) of kinetic Alfvén wave (KAW) turbulence in three spatial dimensions Podesta (2013); Howes (2015).

In a slab equilibrium magnetic geometry (pointing in the $\mathbf{e}_{z}$ direction), a " $\delta f$ " perturbed approach is employed for a constant and local Maxwellian background distribution $F_{\sigma}$ with background density $n_{\sigma}$ and temperature $T_{\sigma}$, around which fluctuations develop in the gyrocenter distribution functions $h_{\sigma}=h_{\sigma}\left(x, y, z, v_{\|}, \mu, t\right)$, where $\mu=m v_{\perp}^{2} / 2 B_{0}$ is the magnetic moment containing the perpendicular velocity information $\left(\mu=v_{\perp}^{2}\right.$ in normalized units) and $\sigma$ is the plasma species index.

The nonlinear gyrokinetic system of equations is solved with the Eulerian code GENE Jenko et al. (2000). Up to a first order in the GK ordering, the non-dimensional GK equations have the form,

$\frac{\partial}{\partial t}\left[h_{\sigma}-q_{\sigma} \bar{\chi}_{\sigma} \frac{F_{\sigma}}{T_{\sigma}}\right]+\left[\mathbf{e}_{z} \times \nabla \bar{\chi}_{\sigma}\right] \cdot \nabla h_{\sigma}+v_{\|} v_{\sigma}^{t h} \frac{\partial h_{\sigma}}{\partial z}=\left[\frac{h_{\sigma}}{\partial t}\right]_{c}$

where the $\left[\frac{h_{\sigma}}{\partial t}\right]_{c}$ term represents the change of $h_{\sigma}$ due to the action of a linearized collision operator (here a linearized Landau-Boltzmann collision operator is being used Navarro et al. (2016)), $v_{\sigma}^{t h}=\sqrt{2 T_{\sigma} / m_{\sigma}}$ is the thermal velocity, $q_{\sigma}$ is the electric charge and $m_{\sigma}$ the particle mass.

The (gyro-averaged) gyrokinetic potential is defined as

$$
\bar{\chi}_{\sigma}=\bar{\phi}_{\sigma}+\frac{\mu}{q_{\sigma}} \bar{B}_{\|, \sigma}-v_{\|} v_{\sigma}^{t h} \bar{A}_{\|, \sigma},
$$

where the overbar refers to a species dependent gyroaverage Merz (2009), while $\phi$ is the first order selfconsistent electrostatic potential, $B_{\|}$is the first order magnetic fluctuation in the parallel direction and $A_{\|}$is the first order magnetic potential in the parallel direction. These fields are obtained from their respective field equations,

$$
\begin{aligned}
\nabla_{\perp}^{2} \phi & =-4 \pi \sum_{\sigma} q_{\sigma} n_{\sigma} \\
\nabla_{\perp}^{2} A_{\|} & =-\frac{4 \pi}{c} \sum_{\sigma} j_{\|, \sigma} \\
B_{\|} & =-\frac{4 \pi}{B_{0}} \sum_{\sigma} p_{\perp, \sigma}
\end{aligned}
$$


for sources $\left(n_{\sigma}, j_{\|, \sigma}, p_{\perp, \sigma}\right)$ obtained from the velocity moments of the perturbed distribution function at the particle position (i.e. $\delta f_{\sigma}=h_{\sigma}-q_{\sigma} \phi \frac{F_{\sigma}}{T_{\sigma}}$ ).

The data used in this work is taken from the simulation presented in Told et al. (2015), and it is briefly summarized in the following: The physical parameters of the simulations are chosen to be close to the solar wind conditions at $1 \mathrm{AU}$, with $\beta_{i}=8 \pi n_{i} T_{i} / B_{0}^{2}=1$ and $T_{i} / T_{e}=1$. Proton and electron species are included with their real mass ratio of $m_{i} / m_{e}=1836$. The electron collisionality is chosen to be $\nu_{e}=0.06 \omega_{A 0}$ (with $\nu_{i}=\sqrt{m_{e} / m_{i}} \nu_{e}$ ), and $\omega_{A 0}$ being the linear frequency of the slowest Alfvén wave in the system. The evolution of the gyrocenter distribution is tracked on a grid with the resolution $\left\{N_{x}, N_{y}, N_{z}, N_{v_{\|}}, N_{\mu}, N_{\sigma}\right\}=$ $\{768,768,96,48,15,2\}$, where $\left(N_{x}, N_{y}\right)$ are the perpendicular, $\left(N_{z}\right)$ parallel, $\left(N_{v_{\|}}\right)$parallel velocity, and $\left(N_{\mu}\right)$ magnetic moment grid points, respectively. This covers a perpendicular dealiased wavenumber range of $0.2 \leq$ $k_{\perp} \rho_{i} \leq 51.2$ (or $0.0047 \leq k_{\perp} \rho_{e} \leq 1.19$ ) in a domain $L_{x}=L_{y}=10 \pi \rho_{i}$, with $\rho_{\sigma}=\sqrt{T_{\sigma} m_{\sigma}} c / e B$. In the parallel direction, a $L_{z}=2 \pi L_{\|}$domain is used, where $L_{\|} \gg \rho_{i}$ is assumed by the construction of gyrokinetic theory. A velocity domain up to three thermal velocity units is taken in each direction. Last, the fluctuations in the system are driven to a steady state via a magnetic antenna potential, which is prescribed solely at the largest scale in accordance with critical balance and evolved in time according to a Langevin equation TenBarge et al. (2014).

\section{DIAGNOSTICS \& MAGNETIC INTERMITTENCY}

While the main purpose of this work is to analyze the intermittency of the distribution functions in phase space, we first introduce the diagnostics employed using the magnetic field fluctuations (denoted by $\mathbf{B}$ ) to provide both an example of our approach and reference existing electromagnetic intermittency works Wan et al. (2012, 2015); Karimabadi et al. (2013); Tenbarge \& Howes (2013); Perri et al. (2012b,a); Osman et al. (2012b,a, 2014b); Sundkvist et al. (2007); Osman et al. (2014a); Hnat et al. (2005); Kiyani et al. (2007); Wan et al. (2016); Coburn et al. $(2014,2015)$. The literature is vast on this subject of magnetic intermittency and we do not attempt to explore it here.

To quantify spatial intermittency in the direction perpendicular to the magnetic guide field, we use $k$ highpass filtered quantities Frisch (1995). For example, the high-pass perturbed magnetic field is simply defined as

$$
\delta \mathbf{B}_{k}^{>}(\mathbf{x})=\int_{|\mathbf{q}|>k} \widehat{\mathbf{B}}(\mathbf{q}, z) e^{i\left(q_{x} x+q_{y} y\right)} d \mathbf{q}
$$

The perpendicular scaling functions of order $p$ are now defined as

$$
S_{p}(k)=\int\left[\left|\delta B_{x, k}^{>}(\mathbf{x})\right|^{p}+\left|\delta B_{y, k}^{>}(\mathbf{x})\right|^{p}\right] d \mathbf{x} .
$$

From this definition we see that the scaling functions are related to the $L^{p}$-space norms $\left(\left[S_{p}(k)\right]^{1 / p}\right)$ for the scale filtered quantities. We use the name scaling functions for $S_{p}(k)$ to differentiate them from the structure functions traditionally defined on field increments. However, their interpretation and use is the same, as we see next.

For turbulence within an ideal inertial range, the scaling functions are expected to scale with $k$ as $S_{p}(k)=$ $C_{p} k^{-\zeta_{p}}$, where $\zeta_{p}=p m+\gamma_{p}$ and the coefficients $\gamma_{p}$ measure the degree of intermittency. In the absence of intermittency, for which the self-similarity of the fields is exact, the anomalous $\gamma_{p}$ coefficients are zero and the scaling of the scaling functions depends only on their order $p$ and the unique scaling factor $m=\zeta_{3} / 3$. In general, the $C_{p}$ and $\zeta_{p}$ coefficients for different orders $p$ do not need to be related to each other (e.g. $\left.\left(C_{p}\right)^{2} \neq C_{2 p}\right)$, nor be universal. While scaling functions can be linked to the energy spectra and the scale flux of energy, the normalized scaling functions, defined as

$$
A_{p}(k)=\frac{S_{p}(k)}{\left[S_{2}(k)\right]^{p / 2}}
$$

are more useful for the study of intermittency. In the absence of intermittency $A_{p}(k)$ is independent of $k$. This can be easily seen for any self-similar scale transformation, e.g. $\delta \mathbf{B}_{\lambda k}^{>}=\lambda^{-m} \mathbf{B}_{k}^{>}$. However, $k$ independence for $A_{p}(k)$ is also observed for a real space Gaussian distribution, which has sometimes led to the mistaken notion that intermittency is a departure from a Gaussian distribution rather than a departure from scale invariance Hnat et al. (2003). For strongly intermittent $k$-intervals, the $A_{p}(k)$ curves exhibit an explosive separation for different orders $p$.

In FIG. 1-a) we depict the tendency for small scale structures to be less volume filling than larger scales, exemplified by the magnetic field. This is the main effect associated with intermittency in real space. In FIG. 1-b) we plot the same data in the same way, except for randomizing the Fourier modes' phases to showcases the importance of phase correlation in the development of intermittency. See Maron \& Goldreich (2001) for a similar depiction of the importance of Fourier phases in MHD turbulence. 

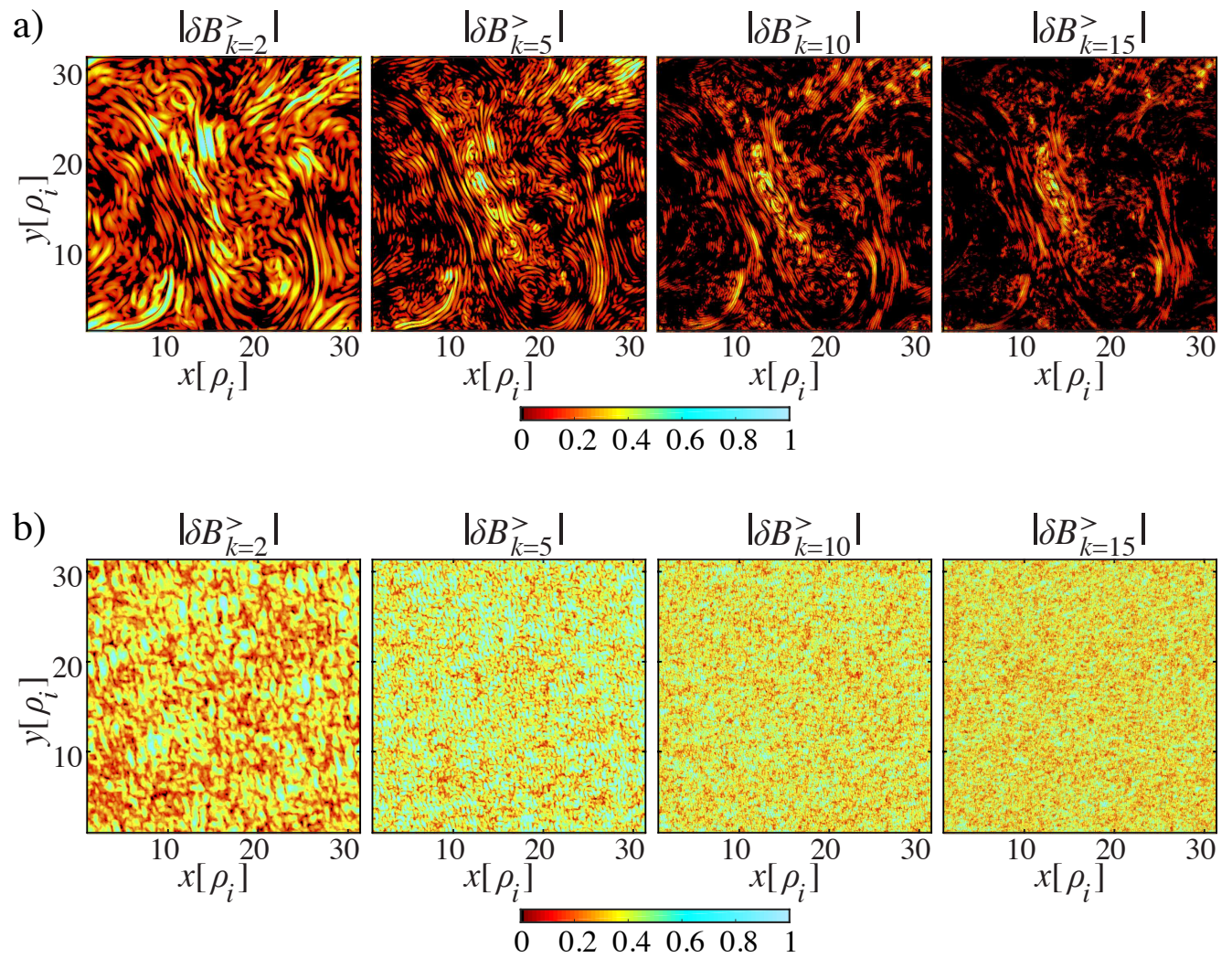

Figure 1. Real space visualization of the norm of perpendicular magnetic fluctuations. In all four panels, the same slice through the $z$ direction is taken, the magnetic field is normalized to its respective maximal value and values less than $0.1 \%$ of the maximum are set to zero (black color). The four panels show the real space data, high-pass filtered beforehand in Fourier space (here $k_{\max }=51$ in units of $1 / \rho_{i}$ ). a) The fact that small scales are less space filling is evident by the progressive increase in the black color. b) We randomized the phases while keeping the same spectral density for the norm of $\delta B_{k}$ to showcase that in the absence of phase correlations we do not have intermittency.

In FIG. 2 we plot the corresponding $A_{p}(k)$ for the magnetic field and the scaling index $\zeta_{p}$, determined by a linear regression of $\log \left(S_{p}\right)=-\zeta_{p} \log (k)+\log \left(C_{p}\right)$ in the interval $k \rho_{i} \in[1,10]$. The qualitative intermittency results found for the magnetic field are consistent with multi-fractal intermittency $\left(\zeta_{p}\right.$ has an increased deviation from the diagonal line for larger $p$ ). Multi-fractal intermittency is typically found for turbulent cascade processes. Here, the kinetic Alfvén wave (KAW) cas-

a)
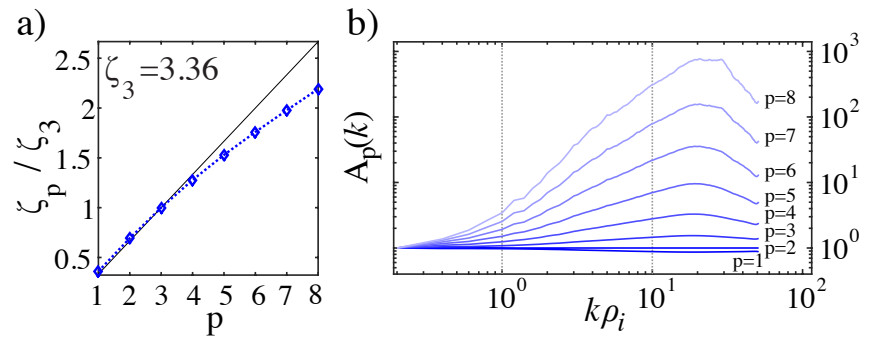

Figure 2. For the perpendicular magnetic field, a) the $\zeta_{p}$ determined by linear regression in the interval delimited by the two dotted lines in the b) plot of $A_{p}(k)$. cade, proceeding at scales smaller than $\rho_{i}$, Howes et al. (2008), Schekochihin et al. (2009), Howes et al. (2011) can be seen as being responsible for the observed multifractal intermittency scaling of the perpendicular magnetic field fluctuations. This emphasizes the importance of nonlinear phase space interaction at kinetic scales. We will return in Section 9 - Discussions and Conclusions to the interpretation of results found for magnetic intermittency and its astrophysical context, after we explore the broader phase space picture.

\section{ON PHASE SPACE MIXING DYNAMICS}

We concentrate hereon the underlying kinetic dynamics. For GK turbulence, the five-dimensional dynamics involve the generation of small scales in the parallel (linear phase mixing Watanabe \& Sugama (2006); Zocco \& Schekochihin (2011); Schekochihin et al. (2016)) and perpendicular (nonlinear phase mixing Tatsuno et al. (2009); Schekochihin et al. (2008); Hammett et al. (1992); Plunk \& Tatsuno (2011); Plunk et al. (2010)) velocity directions. The nonlinear phase mixing responsible for generating structures in the perpendicular 

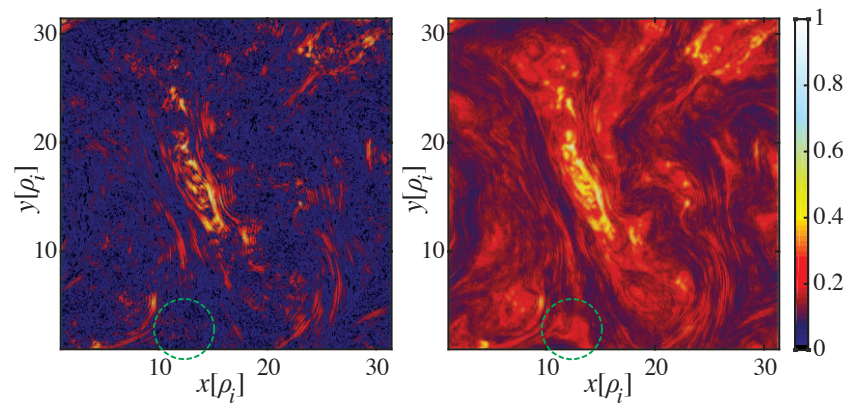

Figure 3. Real space visualization in a $(x, y)$ plane at a fixed $z$ of (left) $\left|\int \delta h_{k=10}^{>} d V_{v}\right|$ and (right) $\int\left|\delta h_{k=10}^{>}\right| d V_{v}$, computed for the electrons, normalized to their respective in-plane maximal value. Example of a structure present in one but not in the other quantity is highlighted by a dotted circle.

velocity direction occurs as part of the same nonlinear interactions that are responsible for the generation of small spatial structures and the emergence of intermittency, namely the advection by drift velocities of the gyro-center distribution function $\left(h_{\sigma}\right)$. Intermittency emerges solely as a result of these nonlinear interactions, however, their balance with linear interactions does matter, as linear terms can suppress or enhance nonlinear couplings. The linear phase mixing term $\left(\sim v_{\|} \partial h_{\sigma} / \partial z\right)$ cannot alone generate intermittency, however, it is the term that leads to linear Landau damping Plunk (2013) (see Villani (2014) for a nonlinear Landau damping treatment) and the generation of ever-smaller parallel velocity structures Chust et al. (2009); Bratanov et al. (2013); Li et al. (2015). In particular, we are concerned in our current work with the parallel velocity scales developed by the electron distribution function (the perpendicular velocity mixing should be small compared with the ion, as $k \rho_{e}<1$ for most of the scales investigated here).

To showcase phase space structures we make use of a simple diagnostic, presented here for the electron species. In FIG. 3 we plot at a given $z$ the $x, y$ density of the integral of the absolute value of $h_{\sigma}$, i.e. $\int\left|\delta h_{k}^{>}\right| d V_{v}$ (which can be seen as giving un upper bound to the fluctuations developed in $h_{\sigma}$ and which exhibits more eddy like structures) and compare it to the absolute value of the integral, i.e. $\left|\int \delta h_{k}^{>} d V_{v}\right|$ (which can be seen as the intensity of the lowest velocity moment), with $d V_{v}=2 \pi B_{0} / m_{\sigma} d v_{\|} d \mu$ the velocity space volume element for the GK problem and with $\delta h_{k}^{>}$defined formally in Eq. (13) later in the article. In the latter case, the velocity space integration naturally leads to cancelations. This happens especially for all odd order moments and, more importantly, for the charge density and electric current from which the self-consistent electromagnetic fields are computed. This simple diagnostic emphasizes the need to analyze kinetic scales turbulence at the level of the distribution functions, when interested in phase space structures, and not over-rely on fluid moments of different orders to avoid inadvertently averaging out dynamical effects.

\section{PARALLEL VELOCITY DECOMPOSITION}

In addition to spatial intermittency, we want to account for structures developed in the parallel velocity for the ion and electron gyrokinetic (non-adiabatic) distribution functions, i.e. $h\left(v_{\|}\right)$, where we suppress dependencies to simplify the notations.

We use a Hermite representation, see Grant and Feix (1967); Armstrong (1967); Hammett et al. (1993) and more recently Hatch et al. (2013, 2014); Loureiro et al. (2013); Numata \& Loureiro (2015); Schekochihin et al. (2016); Servidio et al. (2017), employing the Hermite functions

$$
\psi_{n}\left(v_{\|}\right)=\left(2^{n} n ! \sqrt{\pi}\right)^{-1 / 2} e^{v_{\|}^{2} / 2}\left(-\frac{d}{d v_{\|}}\right)^{n} e^{-v_{\|}^{2}}
$$

which are orthonormal $\int_{-\infty}^{\infty} \psi_{n}\left(v_{\|}\right) \psi_{m}\left(v_{\|}\right) \mathrm{d} v_{\|}=\delta_{n m}$, with $\delta_{n m}$ the Kronecker delta. The $n$-filtered parallel velocity is defined here as

$$
h_{n}\left(v_{\|}\right)=\hat{h}_{n} \psi_{n}\left(v_{\|}\right)
$$

where

$$
\hat{h}_{n}=\int_{-\infty}^{+\infty} h\left(v_{\|}\right) \psi_{n}\left(v_{\|}\right) d v_{\|},
$$

are Hermite amplitudes related to velocity moments of the distribution function; $n=\{0,1,2\}$ relate respectively to fluctuations of the particle density, bulk velocity and particle kinetic energy and are seen as fluid like contributions Loureiro et al. (2013); Numata \& Loureiro (2015); Schekochihin et al. (2016). The original distribution function is simply obtained as a sum over all possible $n$-filtered contributions, i.e.

$$
h\left(v_{\|}\right)=\sum_{n=0}^{\infty} h_{n}\left(v_{\|}\right) .
$$

While $h_{0}\left(v_{\|}\right)$has a simple Gaussian form in $v_{\|}$, for ever larger values of the integer $n$ we select ever smaller scales in $v_{\|}$. Velocity scales represented by $n \geq 3$ can be seen as kinetic only contributions that are not captured by simple fluid closures (e.g. for Grad's 13 moment equations Grad (1949)) and are deemed to have $n$ independent dynamics Parker \& Carati (1995) (i.e. the evolution equations for $n \geq 3$ have the same form). To observe the influence of these kinetic only velocity 
structures, we designate $h_{\geq 3}\left(v_{\|}\right)$as the sum over all $n \geq 3$ contributions, i.e. $h_{\geq 3}\left(v_{\|}\right)=\sum_{n=3}^{\infty} h_{n}\left(v_{\|}\right)=$ $h\left(v_{\|}\right)-h_{0}\left(v_{\|}\right)-h_{1}\left(v_{\|}\right)-h_{2}\left(v_{\|}\right)$.

\section{KINETIC STRUCTURE FUNCTIONS}

The high-pass filters for $h_{\sigma}$, omitting the $\sigma$ species index to simplify the notations, are found as

$$
\delta h_{k}^{>}\left(x, y, z, v_{\|}, \mu\right)=\int_{|\mathbf{q}|>k} \widehat{h}\left(\mathbf{q}, z, v_{\|}, \mu\right) e^{i\left(q_{x} x+q_{y} y\right)} d \mathbf{q} .
$$

The scaling functions of order $p$ for a given species $\sigma$ are defined as

$$
S_{p}(k)=\int\left|\delta h_{k}^{>}\left(x, y, z, v_{\|}, \mu\right)\right|^{p} d V,
$$

where $d V=d x d y d z d V_{v}$ is the five-dimensional phase space volume element. Considering $\left|\delta h_{k}^{>}\right|$ensures that velocity space cancelations do not occur during integration. Here, we take a single given plane in $z$. The definition of $A_{p}(k)$ is still given by Eq. (8), with the same interpretation.

We plot the normalized structure functions $A_{p}(k)$ computed for the ions (protons) in FIG. 4 and for the electrons in FIG. 5. In the panels below each figure we show the $p$-scaling of the exponents $\zeta_{p}$, computed as for the magnetic field in the $k$-intervals separated by vertical dotted lines. Here, we are interested in the qualitative form of the $\zeta_{p} / \zeta_{3}$ curves and a refinement of our choice of

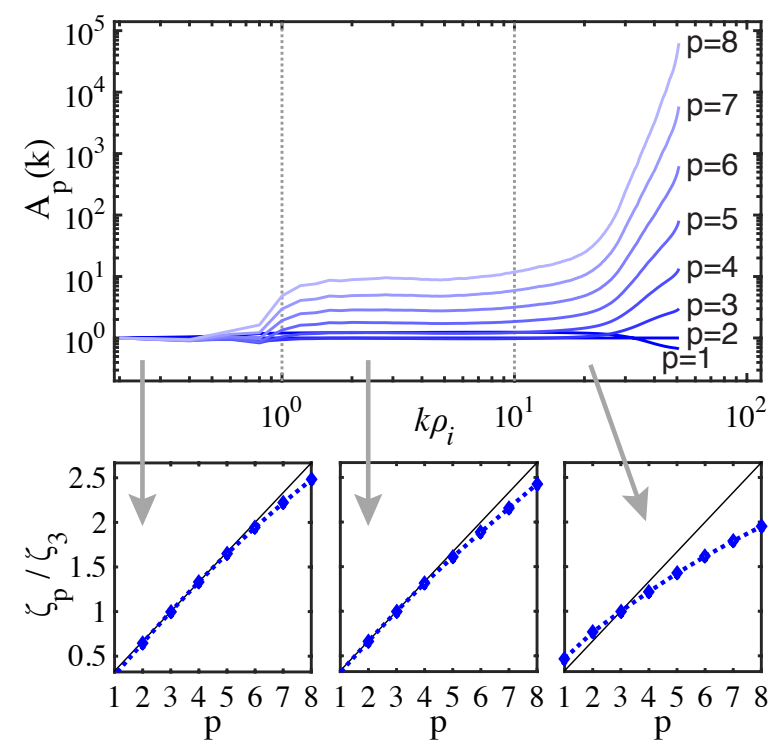

Figure 4. Normalized structure functions of orders $p=$ 1..8, for the ion (proton) distribution function. The curves are normalized to their respective value at $k \rho_{i}=0.2$. The associated panels plot $\zeta_{p}$ determined by linear regression in the intervals indicated.

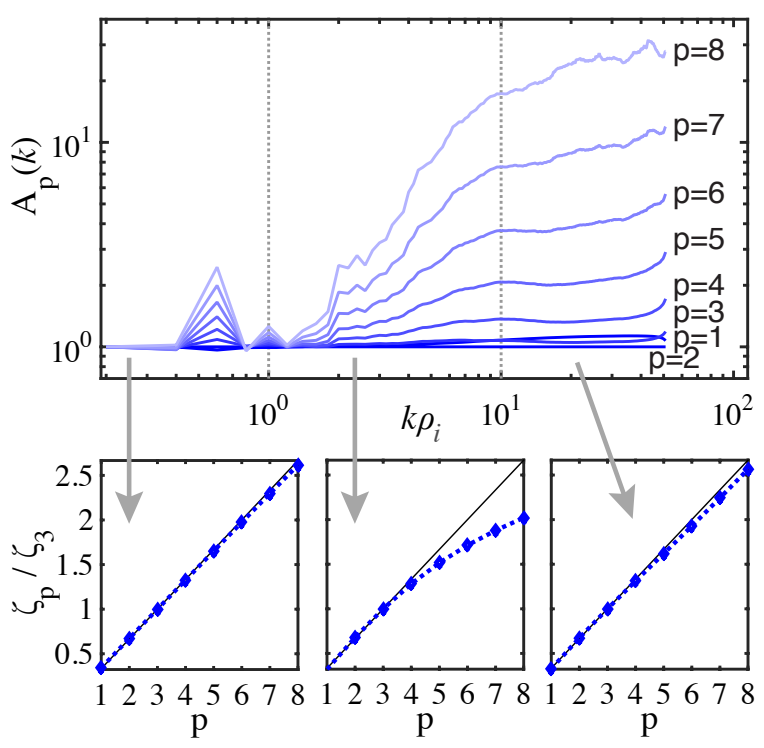

Figure 5. Normalized structure functions of orders $p=1 . .8$, for the electron distribution function. The curves are normalized to their respective value at $k \rho_{i}=0.2$. The associated panels plot $\zeta_{p}$ determined by linear regression in the intervals indicated.
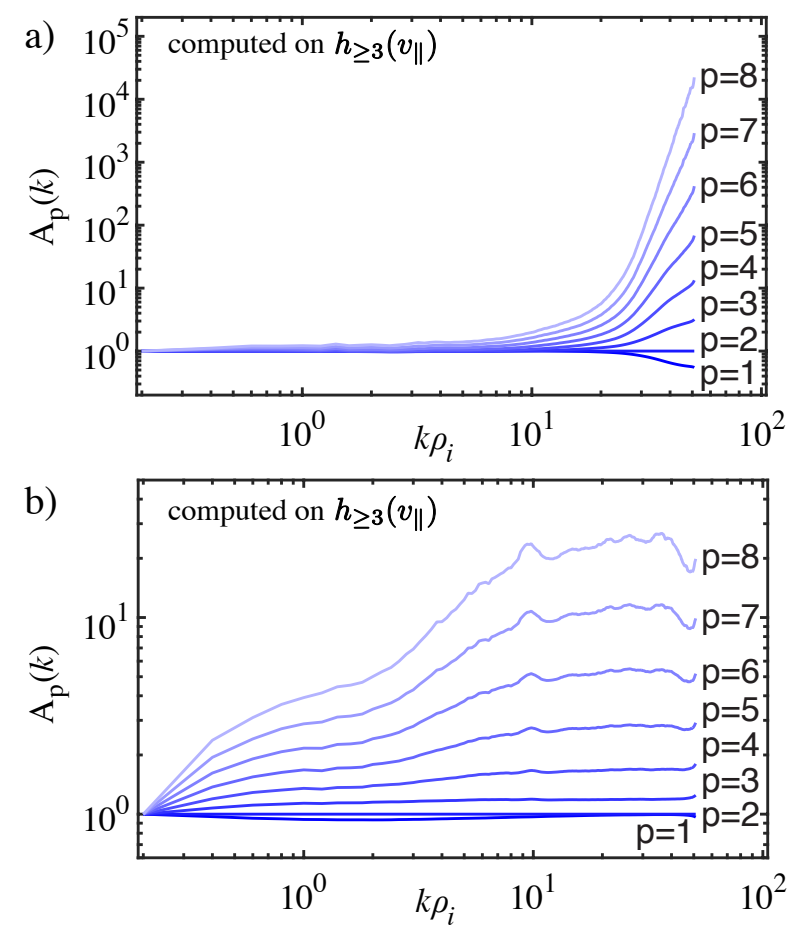

Figure 6. Normalized structure functions of orders $p=1 . .8$, for a) the ion (proton) and b) electron distribution function computed on $h_{\geq 3}\left(v_{\|}\right)=h\left(v_{\|}\right)-h_{0}\left(v_{\|}\right)-h_{1}\left(v_{\|}\right)-h_{2}\left(v_{\|}\right)$.

$k$-intervals does not impact this aspect in a meaningful way.

For the end of the fluid range $\left(k \rho_{i}<1\right)$, we see an absence (or a strong attenuation) of intermittency. In this 


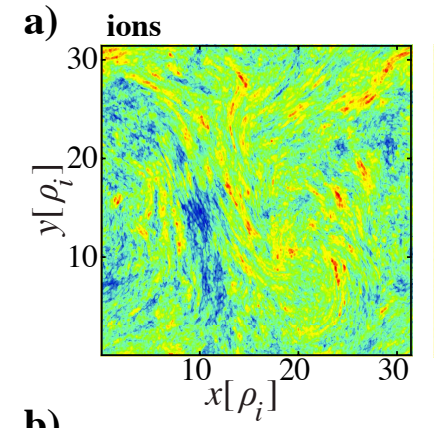

b) ions

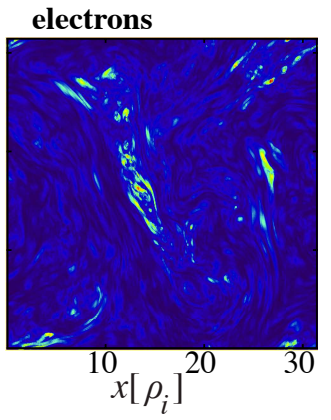

electrons

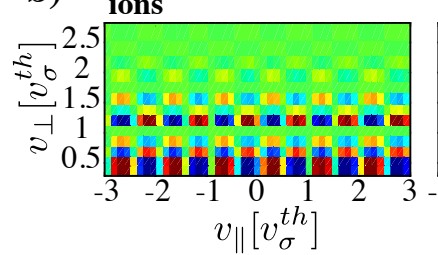

c)

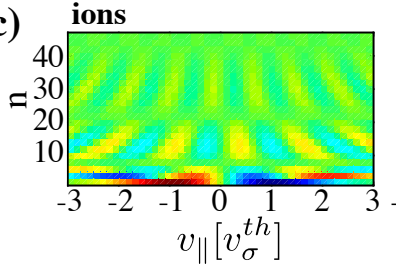

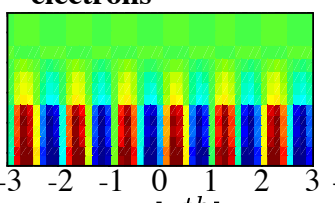

$v_{\|}\left[v_{\sigma}^{t h}\right]$

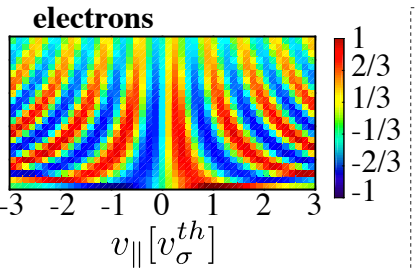

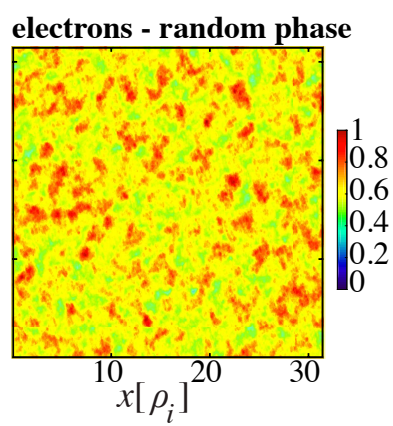

electrons - random phase
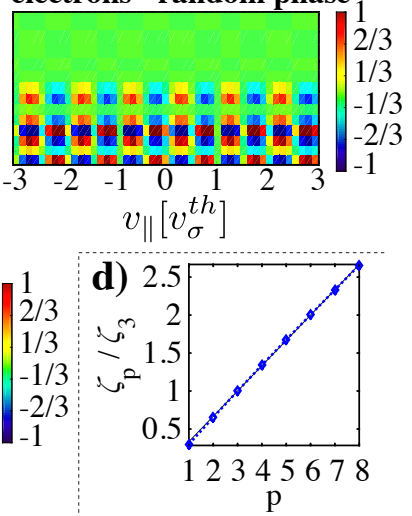

Figure 7. a) Real space visualization of $\int\left|h_{\geq 3}\right|^{2} d V_{v}$ in a $(x, y)$ plane at a given $z$, normalized to their respective in plane maximal value. b) velocity structures for $h_{21}$ at a given spatial point of high intensity in panels-a (coordinates $\{x, y\} \approx\{15,12.5\}$ ), normalized to the maximal $\left\{v_{\|}, v_{\perp}\right\}$ value in each case. c) the $\mu$ integrated $n$-scale structures as a function of $v_{\|}$, i.e. $h_{n}\left(v_{\|}\right)$at the same spatial point as above, normalized to the maximal value in each case. d) the $\zeta_{p}$ scaling for the interval $k \rho_{i} \in[1,10]$ computed for the phase randomized electrons.

range, fluctuations in the electrons' $A_{p}(k)$ are present. In FIG. 5 they take the form of the two small bumps due to the normalization of $A_{p}(k)$ to $A_{p}\left(k_{\min }\right)$ that collapses the curves of different $p$-value. Their increase is not unbounded and they show no impact on the overall intermittency. This is clear from the $\zeta_{p}$ scaling in the respective interval. At $k \rho_{i}=1$ we see a clear sign in the change of the dynamics as the fluid range transitions to the kinetic one.

Considering the kinetic scales $\left(k \rho_{i}>1\right)$, the ions (Fig. 4) show a strong intermittent behavior at the smallest of scales (captured by the divergence of $A_{p}(k)$ curves and the departure of the $\zeta_{p}$ scaling from the nonintermittency line). The electrons (Fig. 5) exhibit a multi-fractal intermittency in the range $k \rho_{i} \in[1,10]$ and a non-intermittent behavior (mono-fractal or at least very weak multi-fractal scaling) at the smallest scales (captured best by the $\zeta_{p}$ scalings). These regimes seem to correspond with the peak of free energy dissipation for each species (see FIG. 4 in Told et al. (2015)). In analogy with classical fluid turbulence, multi-fractal intermittency indicates a local breaking of scale invariance and is to be expected close to dissipation ranges. However, we note that the dissipation is different in nature for the two species, as the electron dissipation is mainly due to parallel velocity collisions Navarro et al. (2016).

In FIG. 6, we show the same analysis performed on the kinetic only velocity scale contributions, given by $h_{\geq 3}\left(v_{\|}\right)$, for which we mention that the corresponding $\zeta_{p}$ scalings remain qualitatively the same. For the ions, looking at the kinetic only velocity contributions $h_{\geq 3}\left(v_{\|}\right)$, we do not see the break in the scaling of $A_{p}$ at $k \rho_{i} \approx 1$. While this break does not influence the anomalous scaling of $\zeta_{p}$, it shows that the non-universal $C_{p}$ coefficients (e.g. $C_{8} \neq C_{4}^{2}$, as $C_{p}$ are not just a function of $p$ ) are sensitive to fluid-like velocity contributions. The fluid-like velocity contributions have a stronger impact on the electrons, attenuating their intermittency at large spatial scales $\left(k \rho_{i}<1\right)$. Considering the absence of a break in the dynamics at $k \rho_{i}=1$ (for ions in particular), we can only conclude that this break in the full distribution is mainly due to fluid moments and the kinetic contributions are mainly insensitive to a transition between different $k \rho_{i}$ scale regimes.

Overall an intriguing picture is formed. Electrons are multi-fractal in a scale range where collisions in the parallel velocity dominate ( $96 \%$ of the total electron dissipation Navarro et al. (2016)), but quickly lose this charac- 
ter past this range, indicating a change in the nature of the subsequent electron cascade towards the smallest of scales (that just "sweeps away" any remaining energy). The presence of strong parallel velocity collisions in a given $k \rho_{i}$ range requires a strong flux of energy in the parallel velocity. This flux is caused by the linear phase mixing, as clearly showcased by Meyrand et al. (2019). We can thus correlate the transition for electrons from a multi-fractal to a mono-fractal scaling at the smallest of scales to the presence or absence of a strong linear phase mixing. We speculate on the implications of phase mixing on intermittency in Section 9 -Discussions and Conclusions.

At the smallest of scales, and only at these scales, the ions (which here exhibit a weak linear phase mixing effect at all scales) are found to be strongly multi-fractal. We know that ions dissipate mainly due to collisions in the perpendicular direction Navarro et al. (2016) and we know that due to nonlinear phase mixing fine structures in the perpendicular velocity generate fine structures in the perpendicular spatial direction as the two are linked Plunk et al. (2010). The amplification of velocity and position space gradients (that localizes ion structures in the perpendicular direction) at an increased rate to achieve energy dissipation is something to be expected. Using the arguments of Schekochihin et al. (2009) and the critical balance condition for the antenna as means to estimate the frequency of the outer scale, we define $k_{\nu_{i}} \rho_{i} \sim\left(\omega_{A 0} / \nu_{i}\right)^{1 / 2}$ as the scale where the transition between the dominance of the nonlinear phase space cascade and the ion collisional dissipation occurs, finding $k_{\nu_{i}} \rho_{i} \approx 26$. This coincides with the change in the nature of intermittency depicted by the ions.

The species dependent velocity space anisotropy, which influences collisional dissipation, could underlie anisotropic heating in astrophysical plasma. Moreover, we see that phase mixing cannot be separated from the analysis of spatial scales, as an enhanced Landau damping route towards parallel dissipation can impact the nature of intermittency of the turbulent cascade, even if it cannot generate it directly.

\section{VELOCITY SPACE STRUCTURES}

Next, keeping only perpendicular spatial scales smaller than the ion gyroradius (i.e. $k \rho_{i}>1$ ), we look at velocity space structures developed by the ions and electrons. In addition, we consider the electron case for which we randomize the Fourier modes' phases in Eq. (13) while keeping the same spectral energy density. By doing so we destroy the nonlinear correlations. In FIG. 7-a) we plot the $\int\left|h_{\geq 3}\right|^{2} d V_{v}$ for the three cases, visually observing that the electrons kinetic structures are highly intermittent compared to the ions and that they occupy a smaller volume. Randomizing the Fourier phases for the electrons destroys their real space structures and suppresses intermittency. This is clearly seen from FIG. 7-d), as the exponent $\zeta_{p}$ computed in the range $k \rho_{i} \in[1,10]$ lost its multi-fractal scaling once the phases are randomised.

For spatial points located in high intensity structures ( $>90 \%$ value in the FIG. 7-a) plots), we look in FIG. 7b) at the velocity space $\left(v_{\|}, v_{\perp}\right)$ for $h_{21}$, see Eq. (10). Selecting only one parallel velocity scale avoids cancellations (or smudging) from taking place during $v_{\|}$integration and provides a much clearer message. The $n=21$ parallel velocity scale determines the structure size in $v_{\|}$ and is here an arbitrary choice. The dominance of nonlinear phase mixing (the case for ions) can be seen as the structures in $v_{\perp}$ become apparent $\left(v_{\perp}=\sqrt{\mu}\right.$ in normalized units). By comparison, the dominance of linear phase mixing for the electrons manifests as the absence of $v_{\perp}$ structures (except for an exponential decay for large values) and the emergence of long structures of given sign. We mention that non-intense points for the electrons in FIG. 7-a) exhibit velocity space structures similar to those seen for the ions, however, with an intensity smaller by one order of magnitude than points exhibiting linear phase mixing. The linear phase mixing role for the electrons is clear from FIG. 7-c), as large scale velocity structures (low $n$ ) are smoothly transformed into smaller scales (here only odd values of $n$ are shown for clarity). We mention that this picture is lost when phases are randomized (in this case, the picture would look qualitatively similar to the figure depicting the ions).

The most striking result (FIG. 7-b), right panel) is observed for the phase randomized electrons, for which the accentuated linear mixing in velocity space is destroyed. The nonlinear phase correlations are responsible for the emergence of intermittent coherent structures, in which linear mixing is found to dominate. This indicates that a balance between the linear phase mixing and the nonlinear interactions emerges at sub-gyroradius scales, as was shown in Hatch et al. (2013, 2014); Tenbarge \& Howes (2013) and proposed by Schekochihin et al. (2016) at $k \rho_{i} \ll 1$ in drift kinetic turbulence. When phases are randomized, the coherent intermittent structures are destroyed and so is the accentuated linear parallel mixing in velocity space at those locations. We emphasize that phase randomization is not creating perpendicular mixing. Destroying the balance between the nonlinear structures and the linear mixing and the suppression of the latter simply allows for the weak perpendicular velocity structure to become apparent. Accounting for the 
results presented, a stronger conjecture can be made regarding the balance between the linear and nonlinear effects: linear phase mixing, which includes Landau damping, dominates in intermittent structures. While evidence to support this is presented in the current work, our conjecture is far from being proven, which will make the object of a future study.

\section{INFLUENCE OF THE VELOCITY SCALES ON THE PERPENDICULAR CASCADE}

In this section we will use the electrons to showcase how velocity scales influence the perpendicular cascade. We mention that for this section alone we used a Hermite polynomial representation Hatch et al. $(2013,2014)$ for the analysis, i.e. $H_{n}\left(v_{\|}\right)$relates to Hermite functions as $H_{n}\left(v_{\|}\right)=\psi_{n}\left(v_{\|}\right) e^{v_{\|}^{2} / 2}\left(\right.$ thus $H_{0}\left(v_{\|}\right) \sim 1, H_{1}\left(v_{\|}\right) \sim v_{\|}$,

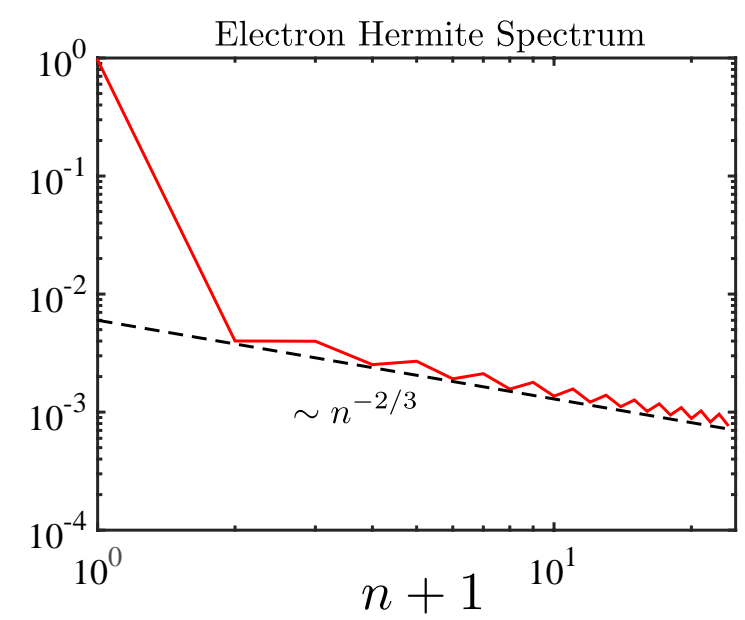

Figure 8. Hermite space spectrum for the electrons. A $n^{-1 / 2}$ scaling is expected for a pure linear Landau damping case and a steeper scaling emerges due to nonlinear decorrelation. The $n=0$ mode is found to dominate.
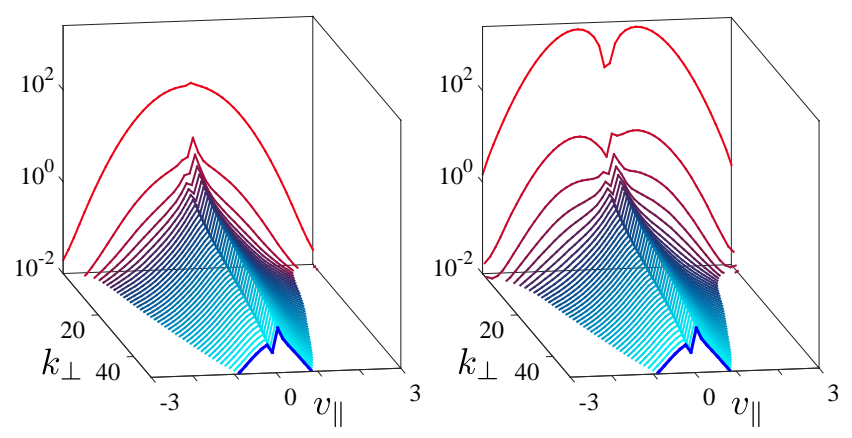

Figure 9. A representation in $\left\{k_{\perp}, v_{\|}\right\}$space for (left) $\left|\delta h_{k_{\perp}}^{>}\left(v_{\|}\right)\right|^{2}$ and for (right) $\left|\delta h_{k_{\perp}}^{>}\left(v_{\|}\right)\left[\delta h_{k_{\perp}}^{>}\left(v_{\|}\right)-q \bar{\chi}_{k_{\perp}}^{>} F / T\right]\right|$ (a proxy for the free energy; it becomes the free energy only once the $v_{\|}$integration has been performed).

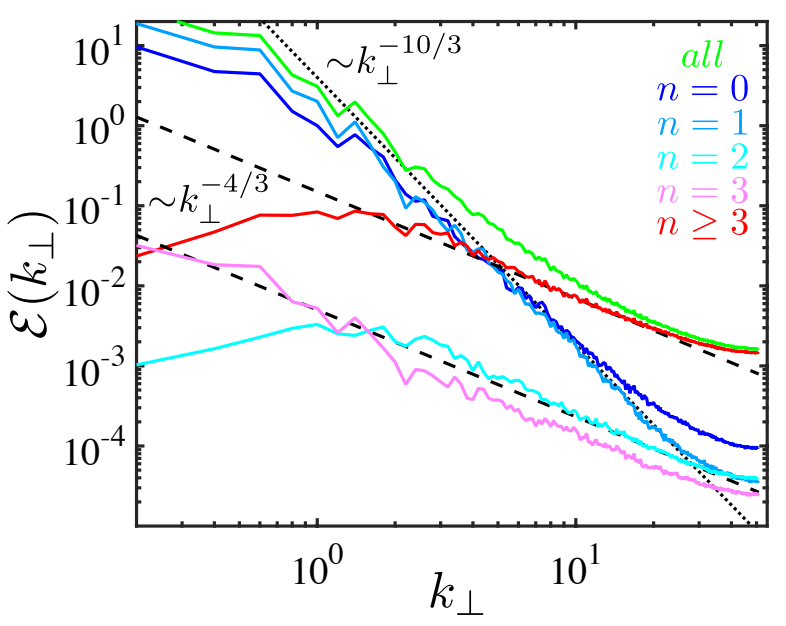

Figure 10. The spectra of the electron contribution to the free energy, $\mathcal{E}=\int d z d v_{\|} d \mu\left(h_{k_{\perp}}\left[h_{k_{\perp}}-q \bar{\chi}_{k_{\perp}} F / T\right]\right)$. Different Hermite modes' contributions are accounted for separately.

etc.). Here,

$$
\begin{gathered}
\tilde{h}_{n}=\int_{-\infty}^{+\infty} h\left(v_{\|}\right) H_{n}\left(v_{\|}\right) d v_{\|} \\
h\left(v_{\|}\right)=\sum_{n=0}^{\infty} \tilde{h}_{n} H_{n}\left(v_{\|}\right) e^{-v_{\|}^{2}} .
\end{gathered}
$$

In FIG. 8, we show the spectrum of $\left|\tilde{h}_{n}\right|^{2}$ for the electrons. Considering that a $n^{-1 / 2}$ scaling is expected for a pure linear Landau damping case Zocco \& Schekochihin (2011), the $-2 / 3$ exponent found indicates that the electrons exhibit a strong linear Landau damping tempered just a little by the nonlinear decorrelation.

While we expect the largest of scales to be dominated by Landau damping, to understand the impact of the cascade we make use of the high-pass filter for $h_{\sigma}$. In FIG. 9, we plot the (high-pass cumulative) spectra of $\left|\delta h_{k_{\perp}}^{>}\left(v_{\|}\right)\right|^{2}$, see Eq. (13), were we integrate over all other directions. For larger values of $k_{\perp}$ we remove more and more the large scale contributions. Concentrating more and more on large $k_{\perp}$ values (thus accounting for more and more of the nonlinear decorrelation at small scales) changes the $v_{\|}$shape of the system (flatter 'tails' compared to the rounder Gaussian bell like shape at low $\left.k_{\perp}\right)$. This is only possible if the system departs further and further from the $n=0$ Hermite contribution in the distribution function (a stronger departure from equilibrium in a way). Here, for the electrons, this change in behavior from the fluid like (low velocity moments) at outer scales to the kinetic like (higher velocity moments) at smaller scales occurs very fast.

This behavior is best seen from FIG. 10, where we plot the (density) $k_{\perp}$ spectrum for the electron component of the free energy and the contribution to this 
spectrum from various $n$ Hermite modes. The scaling of the $n=0,1$ Hermite contributions is steeper compared to the $k_{\perp}$ scaling of the $n \geq 2$. This means that, cumulatively, the $n \geq 2$ contributions will dominate after a certain $k_{\perp}$ value (here $\sim 5$ ). As higher $n$ contributions dominate, we can interpret this as a stronger nonequilibrium state (accentuated departure from a Gaussian distribution) at smaller spatial scales. More importantly, fine parallel velocity structures (corresponding to higher $n$ ) become more significant at smaller $k_{\perp}$ scales. We note that this transition occurs in the interval where the electrons are intermittent. Last, we mention that the $-4 / 3$ and $-10 / 3$ scalings match exactly the values found by Tatsuno et al. (2009) and Plunk et al. (2010) for the scaling of the 'electrostatic' and 'entropy' contributions to free energy in two-dimensional gyrokinetic turbulence (two-dimensional referring to a lack of parallel fluctuations, i.e. lack of $z$ and $v_{\|}$).

\section{DISCUSSIONS AND CONCLUSIONS}

We analyzed large resolution GK simulations, pertinent to kinetic Alfvén wave turbulence. The Alfvén wave turbulent cascade, together with its kinetic continuation at scales smaller than the ion gyroradius, is known to play an important role in the thermalization of ions and electrons in astrophysical plasmas. As kinetic effects such as Landau damping in particular and phase mixing in general become dynamically important and determine the route towards free energy dissipation (entropy production), investigating structures in the broader position-velocity phase space becomes necessary. Restricting the analysis to low-order velocity moments (that give the sources for the electromagnetic fluctuations) is not sufficient to form a complete picture.

At the scale interaction level, intermittency in turbulence manifests itself as an increase in the nonlocal contributions made to the energetic interactions between two scales Laval et al. (2001). From the perspective of scale interactions, the emergence of various degrees of volume filling structures in real space represents an effect of which couplings (local or nonlocal) dominate. Scale locality studies have been performed in the past for GK turbulence Teaca et al. (2012, 2014), including ones Teaca et al. (2017); Told et al. (2015) performed on the same data analyzed here (these studies are available to interested readers, but we do not attempt to link the non-locality and intermittency levels here).

To better understand the self-organization of plasma turbulence at kinetic scales and the emergent intermittency due to the nonlinear interactions, we measured a series of scaling functions directly from the plasma distribution functions. We have found that the electrons are strongly intermittent at kinetic scales $\left(k \rho_{i} \in[1,10]\right)$, while the ions show little to no intermittent behavior in the same range and only show signs of intermittency at much smaller scales $\left(k \rho_{i} \geq 10\right)$. This is not surprising as the electron dissipation peaks in the $k \rho_{i} \in[1,10]$ interval Told et al. (2015) and it is dominated by parallel velocity collisions that require an accentuated linear mixing Navarro et al. (2016), while ions were found to dissipate in the perpendicular direction at predominantly high $k \rho_{i}$.

For the simulations analyzed here $(\beta=1)$, the ion inertial scale corresponds to $\rho_{i}$ in value. At scales smaller than the ion inertial scale, the magnetic field is decoupled from the ions and embedded in the electron flow, exhibiting structures similar to $h_{e}$ and developing a similar multi-fractal intermittent distribution of real space structures as depicted by the electrons. This link includes (but not shown explicitly here) a mono-fractal picture for the magnetic field at scales $k \rho_{i} \geq 10$, as the one exhibited by the electrons.

A mono-fractal scaling is found in solar wind measurements Kiyani et al. (2009) and fluid-kinetic simulations Leonardis et al. (2016) for the smallest scales of magnetic fluctuation $\left(k \rho_{i}>1\right)$. The same mono-fractal scaling is found by us in a range decade smaller compared to the aforementioned studies. We believe the existence of the strong parallel mixing exhibited by the electrons in the $k \rho_{i} \in[1,10]$ range (that imprint their character to the magnetic field) is responsible for our magnetic observations. As Leonardis et al. (2016) is using fluid electrons, this effect cannot be captured by their simulations even if their parameters would support strong parallel mixing. However, their (fluid electrons simulation) agreement with solar wind measurements Kiyani et al. (2009) does give credence to the idea of fluidization of kinetic scale turbulence in some astrophysical plasmas Meyrand et al. (2019). For our discussion, we view the fluidization of kinetic turbulence in its simplest form, that of the perpendicular dissipation channel (which triggers the $k_{\perp}$ cascade) dominating over the parallel velocity one. For us, this is the case for the ions but not the electrons.

For electrons, the region of intermittency observed by us matches the region where linear-mixing dominates Navarro et al. (2016). More correctly, the kinetic region where the fluidization character of the electrons is perturbed by the emergence of a linear Hermite space flux, as presented in Meyrand et al. (2019), corresponds to the observed multi-fractal interval. We speculate that strong linear-mixing triggers multi-fractal intermittency. This could be due to a change in the dimension of the fractal space as the dynamics now fill effectively a larger 
space by populating more $v_{\|}$scales. This may represent a naturally occurring case (but in the opposite direction, increasing rather than decreasing the dimension of the fractal space) as the game presented by Lanotte et al. (2015) for fluid turbulence and deserves a separate investigation.

The absence of this effect in solar wind observations Kiyani et al. (2009) would imply that the MHD cascade continues past the $k \rho_{i}=1$ 'knee' in a (monofractal) kinetic form that preserves a fluid character. Meyrand et al. (2019) qualify their work in the context of accretion disks of highly magnetized plasma and it may be that the pronounced linear mixing can only be observed for strongly magnetized astrophysical plasmas. If an enhanced liner phase mixing can develop without triggering multi-fractal intermittency, or if the two are always linked is an equally important problem. Velocity space measurements that would accompany field measurements in the solar wind Kiyani et al. (2009) would help clarify this aspect for plasma physics in general.

The generation of intermittent structures for the electrons is caused by the same nonlinear term responsible for the cascade to small scales and the perpendicular nonlinear phase mixing in velocity space. Yet, unlike the surrounding background, the most intense and intermittent structures in the electron distribution function exhibit a clear parallel velocity structure, indicative of linear phase mixing (Landau damping) being dominant at those locations (consistent with why magnetic islands are places found to be dominated by Landau damping Loureiro et al. (2013); Numata \& Loureiro (2015)). This shows evidence that nonlinear correlations play a role in supporting a strong linear mixing channel, which is achieved in intermittent structures for the electrons. The existence of a fine parallel velocity structure by itself does not necessarily imply dissipation Hatch et al. (2013, 2014); Plunk \& Parker (2014), nor does it contradict the idea of fluidization of kinetic scale turbulence in astrophysical plasma Meyrand et al. (2019) (as the concept of stochastic echo and indeed the suppression of Landau damping by any mechanism, imply a zero net $n$-flux, not zero $n$ amplitudes). However, the emergence of intermittent structures at kinetic scales that can increase the local linear phase mixing character of the plasma can lead to a drastic point-wise change in the preferred channel for the dissipation of phase space fluctuations. This would change the type of mechanism preferred for the damping of electromagnetic fluctuations in an abrupt way. More work is required in this direction before conclusions can be properly drawn.

Last, an implication to in-situ space plasma measurements can be stated: in addition to the measurement of field fluctuations, particle-field correlation as proposed by Klein \& Howes (2016); Klein et al. (2017); Howes et al. (2017) that can assess velocity space mixing channels are needed to form a complete picture of the physics at kinetic scales. Moreover, reliable pointwise measurements are needed to determine the change in the nature of the preferred dissipation route, parallel or perpendicular to the magnetic guid field, once intermittency emerges.

\section{ACKNOWLEDGMENTS}

We acknowledge the Max-Planck Princeton Center for Plasma Physics for facilitating the discussions that lead to this paper and Greg Hammett in particular for sharing his insight on the problem of plasma turbulence. B. Teaca would like to thank Gregory Eyink for discussions of the topic of turbulence in kinetic plasmas. B. Teaca is partially supported by EPSRC grant No. EP/P02064X/1. The gyrokinetic simulations presented in this work used resources of the National Energy Research Scientific Computing Center, a DOE Office of Science User Facility supported by the Office of Science of the U.S. Department of Energy under Contract No. DE-AC02-05CH11231.

Software: GENE (local version, Jenko et al. (2000)) .

\section{REFERENCES}

Armstrong, T. P. 1967, Phys. Fluids, 10, 1269

Bratanov, V., Jenko, F., Hatch, D., \& Brunner, S. 2013,

Phys. Plasmas, 20, 022108

Brizard, A. J., \& Hahm, T. S. 2007, Rev. Mod. Phys., 79, 421

Bruno, R., \& Carbone, V. 2013, Living Reviews in Solar Physics, 10, doi:10.12942/lrsp-2013-2

Cerri, S. S., Califano, F., Jenko, F., Told, D., \& Rincon, F. 2016, The Astrophysical Journal Letters, 822, L12
Cerri, S. S., Kunz, M. W., \& Califano, F. 2018, The

Astrophysical Journal Letters, 856, L13

Chen, C. H. K., Klein, K. G., \& Howes, G. G. 2019, Nature Communications, 10, 740

Chust, T., Belmont, G., Mottez, F., \& Hess, S. 2009, Phys. Plasmas, 16, 092104

Coburn, J. T., Forman, M. A., Smith, C. W., Vasquez, B. J., \& Stawarz, J. E. 2015, Philosophical Transactions of the Royal Society A: Mathematical, 373, 20140150 
Coburn, J. T., Smith, C. W., Vasquez, B. J., Forman, M. A., \& Stawarz, J. E. 2014, The Astrophysical Journal, 786,52

Eyink, G. L. 2018, Physical Review X, 8, 041020

Frisch, U. 1995, Turbulence, Cambridge University Press

Grad, H. 1949, Comm. Pure Appl. Math., 2, 331

Grant, F. C., \& Feix, M. R. 1967, Phys. Fluids, 10, 696

Hammett, G. W., Dorland, W., \& Perkins, F. W. 1992, Physics of Fluids B, 4, 2052

Hammett, G. W., Beer, M. A., \& Dorland, W., et al. 1993, Plasma Phys. Control. Fusion, 35, 973

Hatch, D. R., Jenko, F., Bratanov, V., \& Navarro, A. B. 2014, J. Plasma Phys., 80, 531

Hatch, D. R., Jenko, F., Navarro, A. B., \& Bratanov, V. 2013, Phys. Rev. Lett., 111, 175001

Hnat, B., Chapman, S. C., \& Rowlands, G. 2003, Physical Review E, 67, 056404

Hnat, B., Chapman, S. C., \& Rowlands, G. 2005, Phys. Rev. Lett., 94, 204502

Howes, G. G., Cowley, S. C., Dorland, W., et al. 2006, The Astrophysical Journal, 651, 590

Howes, G. G., Cowley, S. C., Dorland, W., et al. 2008, J. Geophys. Res., 113, A05103

Howes, G. G., Dorland, W., Cowley, S. C., et al. 2008, Phys. Rev. Lett., 100, 65004

Howes, G. G., Tenbarge, J. M., Dorland, W., et al. 2011, Phys. Rev. Lett., 107, 35004

Howes, G. G. 2015, J. Plasma Phys., 81, 325810203

Howes, G. G., Klein, K. G., \& Li, T. C. 2017, J. Plasma Phys. 83, 705830102

Jenko, F., Dorland, W., Kotschenreuther, M., \& Rogers, B. N. 2000, Phys. Plasmas, 7, 1904

Kanekar, A., Schekochihin, A. A., Dorland, W., \& Loureiro, N. F. 2015, J. Plasma Phys., 81, 305810104

Karimabadi, H., Roytershteyn, V., Wan, M., et al. 2013, Phys. Plasmas, 20, 012303

Kawazura, Y., Barnes, M., \& Schekochihin, A. A. 2019, Proc Natl Acad Sci USA, 116, 771

Kiyani, K., Chapman, S. C., Hnat, B., \& Nicol, R. M. 2007, Phys. Rev. Lett., 98, 211101

Kiyani, K., Chapman, S. C., Khotyaintsev, Yu. V., et al. 2009, Phys. Rev. Lett., 103, 075006

Kiyani, K., Osman, K., \& Chapman, S. 2015, Phil. Trans. R. Soc. A, 373, 2041

Klein, K. G., \& Howes, G. G. 2016, The Astrophysical Journal Letters, 826, 1

Klein, K. G., Howes, G. G., \& TenBarge, J. M. 2016, J. Plasma Phys. 83, 535830401

Krommes, J. A. 2012, Annual Review of Fluid Mechanics, 44,175
Kunz, M. W., Schekochihin, A. A., Chen, C. H. K., et al. 2015, J. Plasma Phys., 81, 325810501

Laval, J.-P., Dubrulle, B., \& Nazarenko, S. 2001, Physics of Fluids, 13, 1995

Leonardis, E., Sorriso-Valvo, L., Valentini, F., et al. 2016, Phys. Plasmas, 23, 022307

Li, T. C., Howes, G. G., Klein, K. G., \& TenBarge, J. M. 2015, arXiv, astro-ph.SR, 1510.02842v1

Loureiro, N. F., Schekochihin, A. A., \& Zocco, A. 2013, Phys. Rev. Lett., 111, 025002

Lanotte, A. S., Benzi, R., Malapaka, S. K. 2013 , et al. 2015, Phys. Rev. Lett., 115, 264502

Mandell, N. R., Dorland, W., \& Landreman, M. 2018, J. Plasma Phys., 84, 905840108

Maron, J., \& Goldreich, P. 2001, The Astrophysical Journal, 554, 1175

Marsch, E. 2006, Living Reviews in Solar Physics, 3

Merz, F. 2009, Ph.D. thesis, Universität Münster

Meyrand, R., Kanekar, A., Dorland, W., \& Schekochihin, A. A. 2019, Proc Natl Acad Sci USA, 116, 1185

Navarro, A. B., Morel, P., Albrecht-Marc, M., et al. 2011, Phys. Rev. Lett., 106, 55001

Navarro, A. B., Teaca, B., Told, D., et al. 2016, Phys. Rev. Lett., 117, 245101

Numata, R., \& Loureiro, N. F. 2015, J. Plasma Phys., 81, 305810201

Osman, K. T., Kiyani, K. H., Chapman, S. C., \& Hnat, B. 2014a, The Astrophysical Journal Letters, 783, L27

Osman, K. T., Matthaeus, W. H., Gosling, J. T., et al. 2014b, Phys. Rev. Lett., 112, 215002

Osman, K. T., Matthaeus, W. H., Hnat, B., \& Chapman, S. C. 2012a, Phys. Rev. Lett., 108, 261103

Osman, K. T., Matthaeus, W. H., Wan, M., \& Rappazzo, A. F. 2012b, Phys. Rev. Lett., 108, 261102

Parker, J. T., \& Dellar, P. J. 2015, Journal of Plasma Physics, 81, 305810203

Parker, S. E., \& Carati, D. 1995, Phys. Rev. Lett., 75, 441

Perri, S., Carbone, V., Vecchio, A., et al. 2012a, Phys. Rev. Lett., 109, 245004

Perri, S., Goldstein, M. L., Dorelli, J. C., \& Sahraoui, F. 2012b, Phys. Rev. Lett., 109, 191101

Plunk, G. G. 2013, Phys. Plasmas, 20, 032304

Plunk, G. G., Cowley, S. C., Schekochihin, A. A., \& Tatsuno, T. 2010, J. Fluid Mech., 664, 407

Plunk, G. G., \& Tatsuno, T. 2011, Phys. Rev. Lett., 106, 165003

Plunk, G. G., \& Parker, J. T. 2014, Eur. Phys. J. D, 68,296

Podesta, J. J. 2013, Solar Physics, 286, 529

Schekochihin, A. A., Cowley, S. C., Dorland, W., et al. 2008, Plasma Phys. Control. Fusion, 50, 4024 
Schekochihin, A. A., Cowley, S. C., Dorland, W., et al. 2009, The Astrophysical Journal Supplement, 182, 310

Schekochihin, A. A., Parker, J. T., Highcock, E. G., et al. 2016, J. Plasma Phys., 82, 905820212

Servidio, S., Chasapis, A., Matthaeus, W., et al. 2017, Phys. Rev. Lett., 119, 205101

Sundkvist, D., Retinò, A., Vaivads, A., \& Bale, S. D. 2007, Phys. Rev. Lett., 99, 025004

Tatsuno, T., Dorland, W., Schekochihin, A. A., et al. 2009, Phys. Rev. Lett., 103, 15003

Teaca, B., Jenko, F., \& Told, D. 2017, New J. Phys., 19, 045001

Teaca, B., Navarro, A. B., \& Jenko, F. 2014, Phys. Plasmas, 21, 072308

Teaca, B., Navarro, A. B., Jenko, F., Brunner, S., \& Villard, L. 2012, Phys. Rev. Lett., 109, 235003

TenBarge, J., Howes, G., Dorland, W., \& Hammett, G. 2014, Computer Physics Communications, 185, 578
Tenbarge, J. M., \& Howes, G. G. 2013, The Astrophysical Journal Letters, 771, L27

Told, D., Cookmeyer, J., Muller, F., Astfalk, P., \& Jenko, F. 2016, New J. Phys., 18, 1

Told, D., Jenko, F., Tenbarge, J., Howes, G., \& Hammett, G. 2015, Phys. Rev. Lett., 115, 025003

Villani, C. 2014, Phys. Plasmas, 21, 0901

Wan, M., Matthaeus, W. H., Roytershteyn, V., et al. 2015, Phys. Rev. Lett., 114, 175002

Wan, M., Matthaeus, W. H., Roytershteyn, V., et al. 2016, Phys. Plasmas, 23, 042307

Wan, M., Matthaeus, W. H., Karimabadi, H., et al. 2012, Phys. Rev. Lett., 109, 195001

Watanabe, T.-H., \& Sugama, H. 2006, Nucl. Fusion, 46, 24

Zhdankin, V., Uzdensky, D. A., \& Boldyrev, S. 2015, Phys. Rev. Lett., 114, 065002

Zhou, Y., Matthaeus, W. H., \& Dmitruk, P. 2004, Rev. Mod. Phys., 76, 1015

Zocco, A., \& Schekochihin, A. A. 2011, Physics of Plasmas, 18,102309 